\title{
2009 Alzheimer Award
}

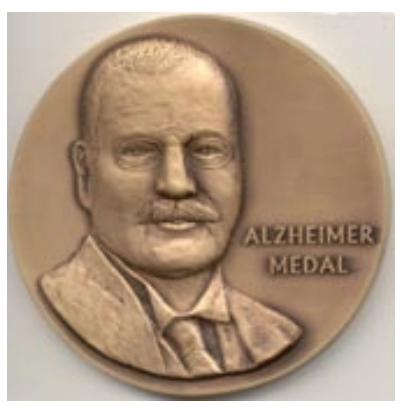

Each year, the Associate Editors of the journal select the best article from the previous year's volumes. The awardee is presented the Alzheimer Medal, a 3" bronze medal with the likeness of Alois Alzheimer. This yearly award is made possible by support from IOS Press and Elan Pharmaceuticals. Through the generous support of Elan, the winner of the 2009 Alzheimer Award will receive a USD7,500 cash prize.

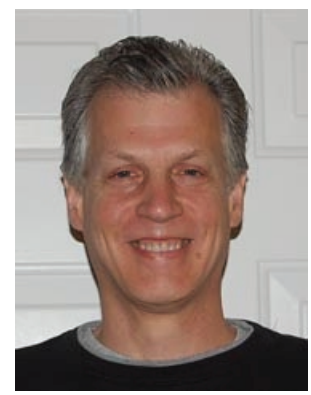

2009 Awardee

John Cogswell, Ph.D.

John Cogswell, Ph.D., a former researcher at GlaxoSmithKline Inc., Research Triangle Park, NC received his initial training from Duke University, Durham, North Carolina followed by postdoctoral fellowships in cellular and molecular immunology at the University of California, Los Angeles and University of North Carolina, Chapel Hill. In 16 years at GSK, John and colleagues developed high content qRT-PCR and histology applications that identified tractable targets from the human genome sequencing project and transcriptional biomarkers that decreased attrition in drug discovery.

\section{Importance of Published Article}

The work published in the paper "Identification of miRNA changes in Alzheimer's disease brain and CSF yields putative biomarkers and insights into disease pathways" (J Alzheimers Dis 14 (2008), 27-41) examines changes in microRNA expression and their role in the sporadic form of the disease. MicroRNAs are small regulatory RNAs that affect many basic cellular and biological processes. They are best known for regulating RNA and protein expression in pathways and networks due to the combinatorial binding potential between microRNAs and their targets. Using a sensitive and specific qRT-PCR technology this paper demonstrates altered expression of specific microRNAs in the brain of Alzheimer's disease (AD) subjects. The changes were both dependent and independent of the pathological changes with some detected quite early in the disease. Many of the microRNAs have functional roles in known AD pathways including neuronal differentiation, glutamate, and innate immunity although the potential combinatorial effects on insulin signaling were noteworthy. Because of the need for diagnostic biomarkers of disease progression this paper additionally examines the microRNAs in the cerebrospinal fluid of late stage AD patients. Again AD-specific changes were detected although here microRNAs with known and potential combinatorial effects on $\mathrm{T}$ lymphocyte signaling and inflammation pathways were identified. Because the CSF contains memory T cells and antigen presenting cells involved in immune-surveillance of the brain, these data suggested that microRNA responses in CSF cells might be sentinels of the disease. More work is required to validate whether these changes are specific to $\mathrm{AD}$, can be used to follow the course of $\mathrm{AD}$ or overlap with other neurodegenerative diseases involving inflammation. However, the data highlight the potential of microRNAs to further understand sporadic Alzheimer's disease and provide accessible biomarkers to aid clinical diagnosis. 